\title{
Chronic Total Occlusion Treatment: Unraveling Intra-Stent Restenosis and Laser Atherectomy through Optical Coherence Tomography Imaging
}

\author{
Luis Augusto Palma Dallan ${ }^{1,2 *}$, Marcelo Harada Ribeiro ${ }^{2}$, Gabriel Tensol Pereira Rodrigues ${ }^{1}$, Armando Vergara- \\ Martel $^{1}$, Elder Iarossi Zago ${ }^{1}$, Vladislav Zimin ${ }^{1}$, Mehmet Elbey ${ }^{1}$, Anas Fares ${ }^{1}$, Chinedu Igwe ${ }^{1}$, Marco Costa ${ }^{1}$, \\ Guilherme Ferragut Attizzani ${ }^{1}$ and Hiram Grando Bezerra ${ }^{1}$
}

${ }^{1}$ Division of Cardiovascular Medicine, Harrington Heart and Vascular Institute, University Hospitals Cleveland Medical Center, USA

${ }^{2}$ InCor - Heart Institute, University of Sao Paulo, Brazil

Submission: August 30, 2019; Published: September 20, 2019

*Corresponding author: Luis Augusto Palma Dallan, Division of Cardiovascular Medicine, Harrington Heart and Vascular Institute, University Hospitals Cleveland Medical Center, 11100 Euclid Avenue, Cleveland, Ohio 44106, USA

\begin{abstract}
Excimer Laser Coronary Atherectomy (ELCA) is an adjuvant procedure to Percutaneous Coronary Intervention (PCI) that aims to properly prepare a coronary lesion for stenting. It is an effective form of atherectomy for complex coronary lesions and plays an important role in the management of lesions seen in cases of in-stent restenosis. We describe the case of a patient with multiple comorbidities who underwent intracoronary Optical Coherence Tomography (OCT) guided percutaneous coronary intervention for the treatment of a Chronic Total Occlusion (CTO) due to In-Stent Restenosis (ISR). Based on OCT imaging, it was possible to observe the mechanism of ISR due to Neointimal Hyperplasia (NIH), to define the anatomy of the lesion, and to optimize PCI. Our strategy was to do appropriate debulking of the neointimal hyperplastic tissue using laser atherectomy, with great results and a successful procedure.

Keywords: Laser atherectomy; Hyperplastic; Dyslipidemia; Hypertension; Myocardial infarction; Coronary artery; Ejection fraction

Abbrevations: ELCA: Excimer Laser Coronary Atherectomy; OCT: Optical Coherence Tomography; CTO: Chronic Total Occlusion; PCI: Percutaneous Coronary Intervention; restenosis; stent expansion; ISR: In-Stent Restenosis; NIH: Neointimal Hyperplasia; LAD: Left Anterior Descending Artery
\end{abstract}

\section{Introduction}

Excimer Laser Coronary Atherectomy (ELCA) is an adjuvant procedure to Percutaneous Coronary Intervention (PCI) that aims to properly prepare a coronary lesion for stenting [1,2]. It is an effective form of atherectomy for complex coronary lesions and plays an important role in the management of lesions seen in cases of in-stent restenosis $[3,4]$.

\section{Case Report}

Patient 69 years old, male, with multiple comorbidities, including dyslipidemia, hypertension and prior inferior wall myocardial infarction, with prior Percutaneous Coronary Intervention (PCI) to the right coronary artery (RCA) in 2014. Evolving with insidious chest pain considered typical angina. Left ventricle ejection fraction 55\%. ECG showing diffuse ventricular repolarization changes, especially in the inferior wall. Elective coronary angiography was performed for angina evaluation.
Patient underwent elective coronary angiography, which showed:

a) The left anterior descending artery (LAD) showed atherosclerosis, but without significant disease (Figure 1, Panel A, black arrow);

b) The left mid to distal circumflex artery (LCx) revealed an $85 \%$ stenosis. (Figure 1, Panel A, blue arrow), Submitted to successful left circumflex PCI using drug-eluting stent (DES) 1 month ago (Figure 1, Panel B, blue arrow);

c) The mid RCA showed a Chronic Total Occlusion (CTO) due to in-stent restenosis (Figure 1, Panel C, white arrow), with poor-developed coronary collaterals, thus escalated elective PCI to this vessel using laser atherectomy guided by OCT (Optical Coherence Tomography) was scheduled. 


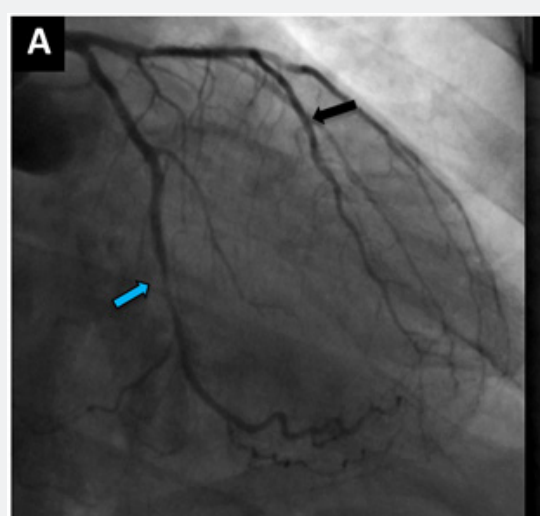

\section{B}

(1)

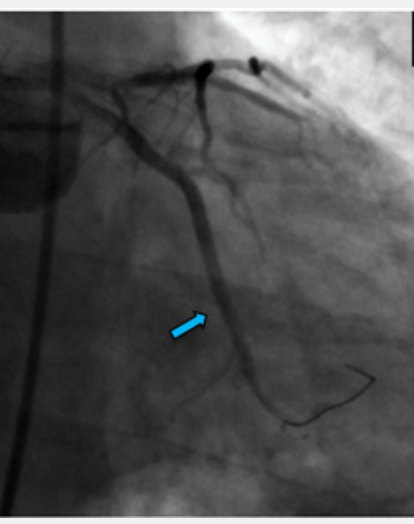

\section{C}

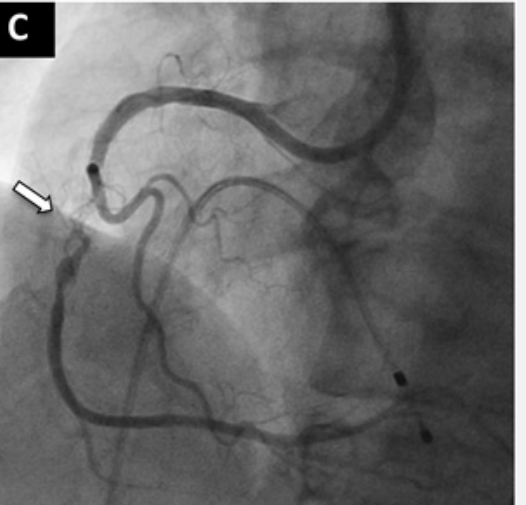

Figure 1: Coronary angiography.

Panel A: Left anterior artery (LAD) without significant lesions (black arrow), and mid left circumflex artery (LCx) with severe $85 \%$ stenosis (blue arrow). Panel B: LCx satisfactory result post stent (blue arrow); Panel C: Mid RCA with chronic total occlusion (white arrow).

The procedure was performed via the right femoral artery. Pre-intervention TIMI flow was 3. PCI was performed within the mid right coronary artery using anterograde approach. RCA was engaged using an AL1 8Fr guiding catheter. After therapeutic anticoagulation, RCA was wired with a Pilot 50 guidewire (Abbott) without difficulty. Pilot wire was exchanged using a Turnpike micro catheter (Vascular Solutions, US) to a BMW guidewire (Abbott).

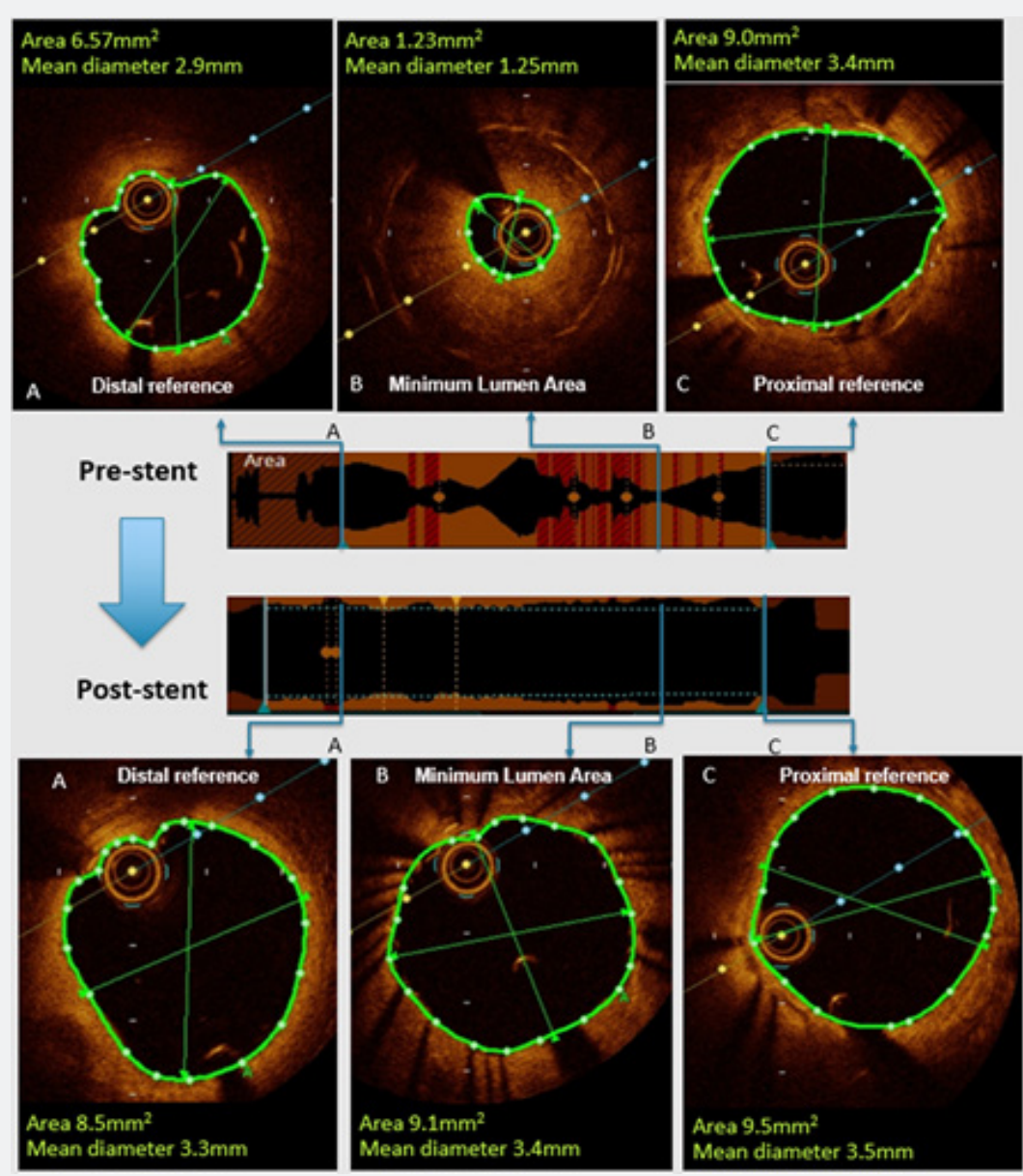

Figure 2: Intravascular Optical Coherence tomography (OCT) showing cross sections in the same region of the minimum luminal area. LPRE-STENT - Panel A: RCA distal reference; Panel B: Mid RCA baseline image revealing neointimal hyperplasia; Panel C: RCA proximal reference. POST-STENT - Panel A: RCA distal reference; Panel B: Mid RCA image revealing great expansion post laser and stent treatment; Panel C: RCA proximal reference. 
Optical Coherence Tomography (OCT) imaging identified a large amount of in-stent fibrotic tissue, corresponding to in-stent restenosis (ISR) due to neointimal hyperplasia (NIH - Figure 2, Panel B pre-stent), and was used for stent sizing and landing zone identification (Figure 2, Panel A and C pre-stent).

Excimer laser coronary atherectomy (ELCA, Philips) using $1.4 \mathrm{~mm}$ was performed (frequency $60 \mathrm{~Hz}$, fluence 40 ), and then escalated to a $2.0 \mathrm{~mm}$ ELCA catheter (frequency $60 \mathrm{~Hz}$, fluence 40 ), with continuous intra-coronary infusion of saline solution. A new OCT imaging pullback showed with significant debulking of neointimal hyperplasia and significant increase in luminal area.

The lesion was predilated with a $3.0 \times 15 \mathrm{~mm}$ non-compliant Euphora balloon (Medtronic) at 16atm. A $3.5 \times 38 \mathrm{~mm}$ Xience Sierra DES (Abbott) was deployed at $14 \mathrm{~atm}$ and post dilated with a $3.5 \times 15 \mathrm{~mm}$ non-compliant Euphora balloon (Medtronic) at 24atm. Final OCT imaging showed adequate stent apposition and expansion (88\%), with no evidence of dissections. (Figure 2, Panel A, B and C post-stent). Final angiogram with excellent angiographic results and final TIMI 3 flow. (Figure 3, Panel D, white arrow).

\section{Discussion}

Laser excimer coronary atherectomy (ELCA) is an adjuvant therapy to percutaneous coronary intervention (PCI), with established safety and efficacy, especially for the treatment of complex lesions requiring neointimal debulking. In this type of atherectomy, a standard 0.014 " guidewire is used over which the $8 \mathrm{~F}$ diameter ELCA catheter is passed for laser application to the obstructive plate region [5].

In our case report, OCT was especially important in determining the restenosis mechanism, lesion anatomy, and procedure planning. We observed ISR with excessive fibrotic tissue inside the previous stent, corresponding to neointimal hyperplasia, with the possibility of failure in the attempt to compact this thick proliferative tissue only by catheter balloon predilation. Thus, OCT imaging guided appropriate neointimal plaque debulking through ELCA and adequate vessel sizing measurements for optimal stent implantation, with successful results.

The importance of debulking is not only due to direct luminal gain, but also to the decrease in the mass of neointimal hyperplasia to be compressed and a greater radial balloon force on the previously implanted stent, allowing for optimal expansion. In summary, a lumen gain was observed by 3 different mechanisms: 1) Ablation of neointimal hyperplasia; 2) Previous stent expansion; 3) Compression of residual neointimal hyperplasia (Figure 3, Panel A, B \& C).
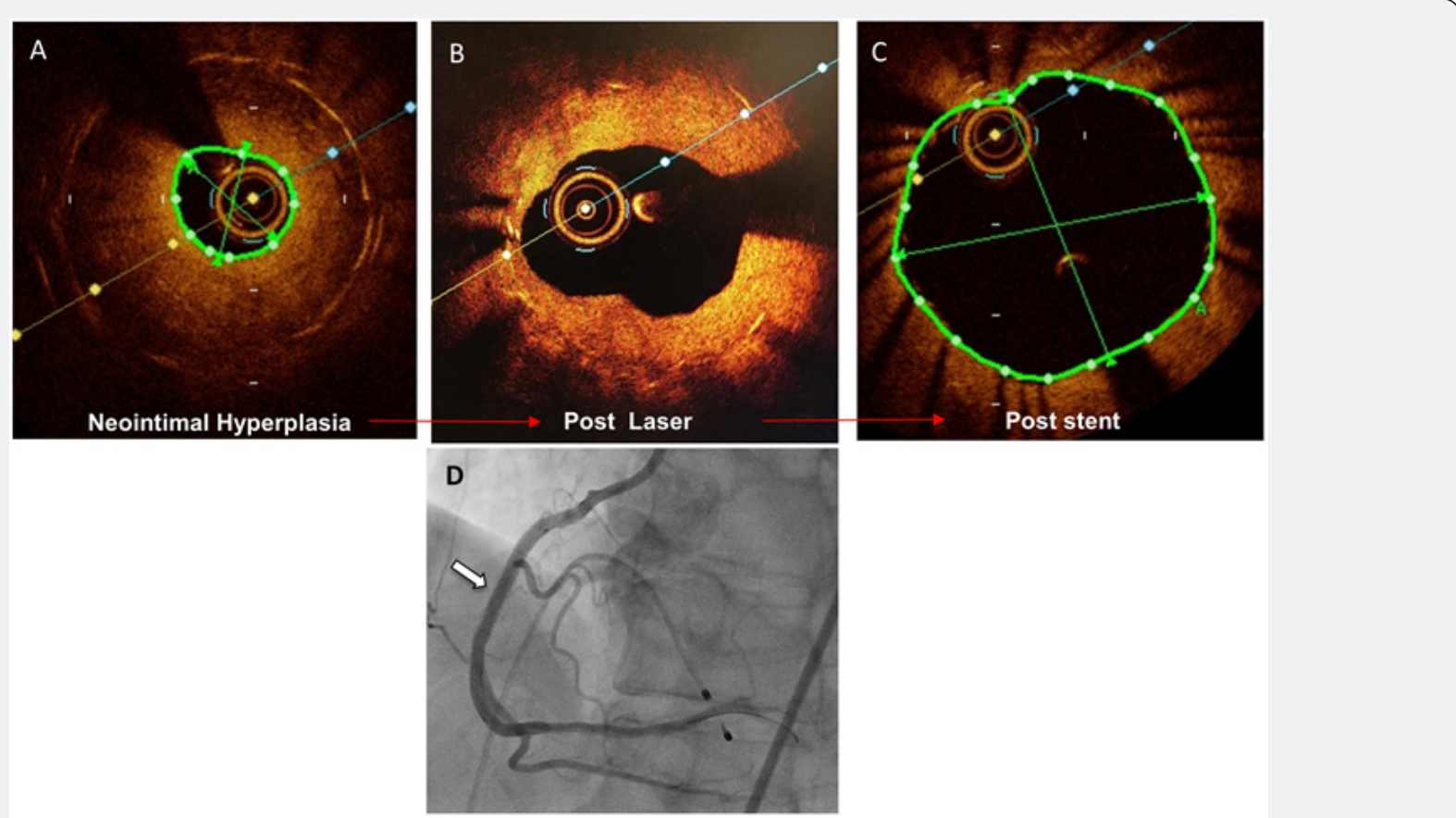

Figure 3: Intravascular Optical Coherence tomography (OCT) showing cross sections in the same region of the minimum luminal area. Panel A: Baseline image revealing neointimal hyperplasia. Panel B: Aspect of the lesion after laser atherectomy. Panel C: Final aspect of the lesion after stenting. Panel D: Coronary angiography showing successful final result of mid RCA treatment.

Currently, ELCA is performed in procedures requiring appropriate lesion debulking, such as acute coronary syndromes, in-stent restenosis, saphenous vein grafts, chronic total occlusions, and calcified lesions [2,3]. The main complications of ELCA are similar to those found in other atherectomy modalities. Specific problems may arise if saline irrigation is interrupted or residual 
contrast is present, which can generate excessive heat and increase the risk of coronary perforation. The CTO treatment procedural limitations are subintimal long lengths with absence of contrast distal to the lesion, implying non-analyzable poor-quality imaging and possible misinterpretation [2,6]. In such cases, predilation would allow increased contrast flow and enhanced imaging.

Lastly, the main limitation of ELCA is the presence of extensive calcification. However, a hybrid procedure can be performed when rotational atherectomy is required but cannot be performed due to the inability of the dedicated guidewire to pass through. In these cases, the ELCA can create a channel to allow the passage of this dedicated guidewire, and thus can be associated with rotational atherectomy, known as the "RASER" technique $[2,6]$.

\section{References}

1. Dallan LAP, Tensol Rodrigues Pereira G, Alaiti MA, Zimin V, VergaraMartel A, et al. (2019) Laser Imaging: Unraveling Laser Atherectomy Mechanisms of Action with Optical Coherence Tomography. Current Cardiovascular Imaging Reports 12.
2. Rawlins J, Din JN, Talwar S, O'Kane P (2016) Coronary Intervention with the Excimer Laser: Review of the Technology and Outcome Data. Interv Cardiol 11(1): 27-32.

3. Dallan LAP, Pereira GTR, Bezerra HG (2019) Management of in-stent restenosis of the left main coronary artery with laser atherectomy guided by intracoronary optical coherence tomography. J Transcat Intervent 27.

4. Nishino M, Mori N, Takiuchi S, Shishikura D, Doi N, et al. (2017) Indications and outcomes of excimer laser coronary atherectomy: Efficacy and safety for thrombotic lesions-The ULTRAMAN registry. J Cardiol 69(1): 314-319.

5. Badr S, Ben-Dor I, Dvir D, Barbash IM, Kitabata H, et al. (2013) The state of the excimer laser for coronary intervention in the drug-eluting stent era. Cardiovasc Revasc Med 14(2): 93-98.

6. Fernandez JP, Hobson AR, McKenzie D, Shah N, Sinha MK, et al. (2013) Beyond the balloon: excimer coronary laser atherectomy used alone or in combination with rotational atherectomy in the treatment of chronic total occlusions, non-crossable and non-expansible coronary lesions. EuroIntervention 9(2): 243-250.

\section{Your next submission with Juniper Publishers will reach you the below assets}

- Quality Editorial service

- Swift Peer Review

- Reprints availability

- E-prints Service

- Manuscript Podcast for convenient understanding

- Global attainment for your research

- Manuscript accessibility in different formats

( Pdf, E-pub, Full Text, Audio)

- Unceasing customer service

Track the below URL for one-step submission https://juniperpublishers.com/online-submission.php 